\title{
Uncertainty propagation in stochastic fractional order processes using spectral methods: A hybrid approach
}

\author{
Pham Luu Trung Duong, Moonyong Lee* \\ School of Chemical Engineering, Yeungnam University, Gyeongsan 712-749, Republic of Korea
}

\section{A R T I C L E I N F O}

\section{Article history:}

Received 1 August 2011

Received in revised form 23 January 2012

Accepted 31 January 2012

Available online 9 February 2012

\section{Keywords:}

Block pulse functions

Fractional calculus

Operational matrix

Stochastic collocation

Polynomial chaos

\begin{abstract}
A B S T R A C T
Stochastic spectral methods are widely used in uncertainty propagation thanks to its ability to obtain highly accurate solution with less computational demand. A novel hybrid spectral method is proposed here that combines generalized polynomial chaos (gPC) and operational matrix approaches. The hybrid method takes advantage of gPC's efficient handling of large parameter uncertainties and overcomes its limited applicability to systems with relatively highly correlated inputs. The hybrid method's use of operational matrices allows analyses of systems with low input correlations without suffering its restriction to small parameter uncertainties. The hybrid method is aimed to propagate uncertainties in fractional order systems with random parameters and random inputs with low correlation lengths. It is validated through several examples with different stochastic uncertainties. Comparison with Monte Carlo and gPC demonstrates the superior computational efficiency of the proposed method.
\end{abstract}

(C) 2012 Elsevier B.V. All rights reserved.

\section{Introduction}

Fractional calculus has applicability across disciplines such as physics, biology, chemistry, finance, physiology, and control engineering [1-5]. It is also recommended for the modeling and identification of flexible structures [6], anomalous system [7], and viscoelastic materials [8] for which integer order systems cannot give accurate results. More details and history about fractional order differential equations can be found in [9-12]. Fractional order systems are often studied using models with fixed deterministic parameters and inputs; however, systems often suffer noise that causes fluctuations in their behavior, making it deviate from deterministic fractional differential models. Therefore, the development of methods capable of characterizing fractional order systems with uncertainties is necessary.

Monte Carlo (MC) is a commonly used probabilistic method of uncertainty quantification [13,14]. It involves the generation of independent realizations of random inputs based on their prescribed probability distribution. For each realization the data are fixed and the problem becomes deterministic. Solving the multiple deterministic realizations builds an ensemble of solutions, i.e. realizations of the random solutions, from which statistical information can be extracted, e.g. mean and variance. It is simple to apply, only involving repeated deterministic simulations; however, convergence is slow and large numbers of calculations are typically needed. For example, mean values typically converge as $1 / \sqrt{M}$, where $M$ is the number of realizations.

Generalized polynomial chaos (gPC) [15-17] is a more recent approach to quantifying uncertainty within system models. It involves expressing stochastic quantities as orthogonal polynomials of the random input parameters; various orthogonal polynomials can be chosen to achieve better convergence. The gPC expansion is essentially a spectral representation in

\footnotetext{
* Corresponding author. Tel.: +82 53810 3241; fax: +82 538113262

E-mail address: mynlee@yu.ac.kr (M. Lee).
} 
random space. It converges quickly when the expanded function depends smoothly on the random parameters. However, the random inputs of many systems involve random processes approximated by truncated Karhunen-Loeve (KL) expansions and the input's dimensionality depends on the correlation lengths of these processes. For input processes with low correlation lengths, the number of dimensions required for accurate representation can be extremely large. The operational matrix method [17] is an alternative approach for quantifying uncertainty in integer order systems, where a system is described by a stochastic operator (operational matrix). It includes Neumann expansions, which express the inverses of stochastic operators in Neumann series. It is most effective for systems with inputs with low correlation lengths, but is restricted to small parameter uncertainties.

Recently, there are several works about quantifying uncertainties for fractional order system [18-20]. However, they considered only a specific type of additive input uncertainties (ideal white noise) without considering uncertainties in the system's parameters. This work proposes a novel hybrid algorithm that combines operational matrices and polynomial chaos to quantify uncertainties in linear fractional order systems with random parameters and random inputs of relatively small correlation length. The stochastic operator is approximated using polynomial chaos instead of Neumann series. This method gives algebraic relationships between the first and second order stochastic moments of a system's input and output, hence bypassing the KL expansions that can require large dimensions for accurate results. It also is not limited by the magnitude of the uncertainty as the traditional operational matrix method is.

Section 2 briefly introduces fractional order systems and the operational matrix technique for uncertainty quantification in fractional order systems, leading to the computation of random matrices' moments. Section 3 demonstrates calculation of the moments of the random matrices with the aid of stochastic collocation. It also outlines MC, operational matrices with Neumann series and gPC to show the limitations of the existing methodologies. Section 4 considers examples to demonstrate the use of the proposed hybrid method. The proposed method is also compared with traditional MC and gPC.

\section{Fractional order system}

\subsection{Governing equation for system dynamics}

Fractional calculus is the generalization of integration and differentiation to consider fundamental operators of non-integer order [21]. Among the several formulations of the generalized derivative, the Riemann-Liouville definition is most commonly used:

$$
D_{0}^{\alpha} f(t)=\frac{1}{\Gamma(m-\alpha)}\left(\frac{d}{d t}\right)^{m} \int_{0}^{t} \frac{f(\tau)}{(t-\tau)^{1-(m-\alpha)}} d \tau
$$

where $\Gamma(x)$ denotes the gamma function; $m$ is the integer satisfying $m-1<\alpha<m ; \alpha>0$ is the real order derivative.

The Riemann-Liouville fractional integral of a function $f(t)$ is defined by:

$$
I_{0}^{\alpha} f(t)=\frac{1}{\Gamma(\alpha)} \int_{0}^{t} \frac{f(\tau)}{(t-\tau)^{1-\alpha}} d \tau
$$

The Laplace transform for a fractional order derivative under zero initial conditions is defined as:

$$
L\left\{D_{0}^{\alpha} f(t)\right\}=s^{\alpha} F(s)
$$

Thus, a fractional order single input single output (SISO) system can be described by the fractional order differential equation:

$$
a_{0} D_{0}^{\alpha_{0}} y(t)+a_{1} D_{0}^{\alpha_{1}} y(t)+\cdots+a_{l} D_{0}^{\alpha_{l}} y(t)=b_{0} D_{0}^{\beta_{0}} u(t)+b_{1} D_{0}^{\beta_{1}} u(t)+\cdots+b_{m} D_{0}^{\beta_{m}} u(t)
$$

or by the transfer function:

$$
G(s)=\frac{Y(s)}{U(s)}=\frac{b_{m} s^{\beta_{m}}+\cdots+b_{0} s^{\beta_{0}}}{a_{l} s^{\alpha_{1}}+\cdots+a_{0} s^{\alpha_{0}}}
$$

where $\alpha_{i}$ and $\beta_{i}$ are arbitrary real positive numbers; and $u(t)$ and $y(t)$ are the system's input and output, respectively.

\subsection{Block pulse operational matrices for fractional order systems}

Block pulse functions constitute a complete set of orthogonal functions and are defined over the time interval [0, $\tau]$ :

$$
\psi_{i}= \begin{cases}1 & \frac{i-1}{N} \tau \leqslant t \leqslant \frac{i}{N} \tau \\ 0 & \text { elsewhere }\end{cases}
$$

where $N$ is the number of block pulse functions.

Thus, any function that can be absolutely integrated on the time interval $[0, \tau]$ can be expanded into a series from the block pulse basis: 


$$
f(t)=\psi_{N}(t)^{T} C_{f}=\sum_{i=1}^{N} c_{f_{i}} \psi_{i}(t)
$$

The expansion coefficients are evaluated as:

$$
c_{f_{i}}=\frac{N}{\tau} \int_{[(i-1) / N] \tau}^{(i / N) \tau} f(t) \psi_{i}(t) d t
$$

Furthermore, any function $g\left(t_{1}, t_{2}\right)$ absolutely integrable on the time interval $[0, \tau] \times[0, \tau]$ can be expanded as:

$$
g\left(t_{1}, t_{2}\right)=\sum_{i=1}^{N} \sum_{j=1}^{N} c_{i j} \psi_{i}\left(t_{1}\right) \psi_{j}\left(t_{2}\right)=\psi^{T}\left(t_{1}\right) C_{g} \psi\left(t_{2}\right)
$$

with coefficient matrix $C_{g}$ defined as:

$$
C_{g}=\left[c_{i j}\right]_{i, j=1}^{N}=\left[\begin{array}{ccc}
c_{11} \cdots & c_{1 j} \cdots & c_{1 N} \\
\vdots & \vdots & \vdots \\
c_{i 1} \cdots & c_{i j} \cdots & c_{i N} \\
\vdots & \vdots & \vdots \\
c_{N 1} \cdots & c_{N j} \cdots & c_{N N}
\end{array}\right] ; \quad c_{i j}=\left(\frac{N}{\tau}\right)^{2} \int_{[(i-1) / N] \tau}^{(i / N) \tau} \int_{[(i-1) / N] \tau}^{(i / N) \tau} g\left(t_{1}, t_{2}\right) \psi_{i}\left(t_{1}\right) \psi_{j}\left(t_{2}\right) d t_{1} d t_{2}
$$

Eq. (2) can be expressed in terms of the operational matrix [22]:

$$
I_{0}^{\alpha} f(t)=\psi(t)^{T} A_{\alpha} C_{f}
$$

where the generalized operational matrix integration of the block pulse function, $A_{\alpha}$, is:

$$
A_{\alpha}=P_{\alpha}^{T}=\left(\frac{\tau}{N}\right)^{\alpha} \frac{1}{\Gamma(\alpha+2)}\left(\begin{array}{ccccc}
f_{1} & f_{2} & f_{3} & \ldots & f_{N} \\
0 & f_{1} & f_{2} & \ldots & f_{N-1} \\
\vdots & \ddots & \ddots & \ddots & \vdots \\
0 & \ldots & \ldots & \cdots & f_{1}
\end{array}\right)^{T}
$$

The elements of the generalized operational matrix integration are given by:

$$
f_{1}=1 ; \quad f_{p}=p^{\alpha+1}-2(p-1)^{\alpha+1}+(p-2)^{\alpha+1} \text { for } p=2,3 \ldots
$$

The generalized operational matrix of a derivative of order $\alpha$ is:

$$
D_{\alpha} A_{\alpha}=I
$$

where $I$ is the identity matrix.

Similarly, the Riemann-Liouville fractional derivative given in (1) can also be expressed in terms of the operational matrix:

$$
D_{0}^{\alpha} f(t)=\psi(t)^{T} D_{\alpha} C_{f}
$$

Thus, the fractional order system in (4) can be rewritten in terms of the operational matrix $A_{G}$ :

$$
A_{G}=\left(a_{l} D_{\alpha_{l}}+\cdots+a_{0} D_{\alpha_{0}}\right)^{-1}\left(b_{m} D_{\beta_{m}}+\cdots+b_{0} D_{\beta_{0}}\right)
$$

The input and output are related by:

$$
C_{Y}=A_{G} C_{U} ; \quad Y(t)=\left(C_{Y}\right)^{T} \psi(t) ; \quad U(t)=\left(C_{U}\right)^{T} \psi(t)
$$

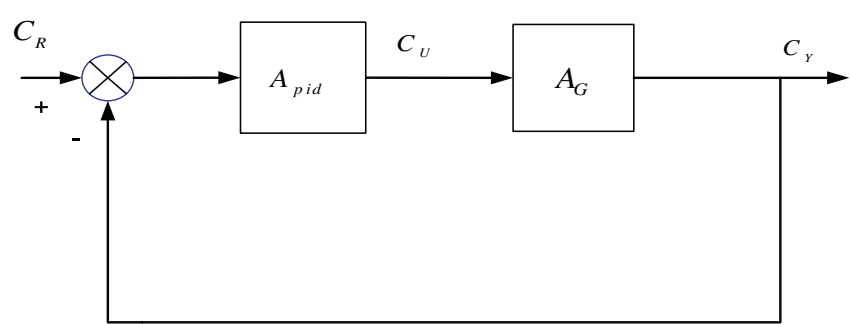

Fig. 1. A closed-loop control system in terms of operational matrices. 
Closed-loop control systems normally comprise several elements, such as the controller and plant in Fig. 1 in terms of the operational matrices. A closed-loop system's operational matrix can be found using block diagram algebra similar to the block algebra used for the transfer function [14].

\subsection{Stochastic analysis of fractional order systems}

Consider the closed-loop system in Fig. 1 that has its input and output linked by:

$$
\begin{aligned}
& C_{Y}=A_{c} C_{R} \\
& R(t)=\psi(t)^{T} C_{R} ; \quad Y(t)=\psi(t)^{T} C_{Y}=\psi(t)^{T} A_{c} C_{R}
\end{aligned}
$$

where $A_{c}$ is the closed-loop system's operational matrix. The input and parameters are random.

Hence, the mean of the input and the output in (19) is calculated as:

$$
m_{R}(t)=E[R(t)]=\psi(t)^{T} E\left[C_{R}\right]=\psi(t)^{T} C_{m_{R}} ; \quad m_{Y}(t)=E[Y(t)]=\psi(t)^{T} E\left[C_{Y}\right]=\psi(t)^{T} C_{m_{Y}}=\psi(t)^{T} E\left[A_{c} C_{R}\right]
$$

where $E[]$ denotes the expectation operator; $C_{m_{R}}=E\left[C_{R}\right] ; C_{m_{Y}}=E\left[C_{Y}\right]$.

The statistical independence of $A_{C}$ and $C_{R}$ leads to:

$$
m_{Y}(t)=\psi(t)^{T} C_{m_{Y}}=\psi(t)^{T} E\left[A_{c}\right] C_{m_{R}}
$$

Thus, the spectral characteristics (or expansion coefficients) of the mathematical expectations of input and output are related by:

$$
C_{m_{Y}}=E\left[A_{c}\right] C_{m_{R}}
$$

Introducing the system's signal in the form of (7) leads to the equation defining the correlation function of the output to be written as:

$$
\theta_{Y Y}\left(t_{1}, t_{2}\right)=E\left[Y\left(t_{1}\right) Y\left(t_{2}\right)\right]=E\left[\psi\left(t_{1}\right)^{T} C_{Y} C_{Y}^{T} \psi\left(t_{2}\right)\right]=\psi\left(t_{1}\right)^{T} E\left[C_{Y} C_{Y}^{T}\right] \psi\left(t_{2}\right)=\psi\left(t_{1}\right)^{T} E\left[A_{c} C_{R}\left(C_{R}\right)^{T} A_{c}^{T}\right] \psi\left(t_{2}\right)
$$

Thus, Eq. (23) becomes:

$$
\theta_{Y Y}\left(t_{1}, t_{2}\right)=\psi\left(t_{1}\right)^{T} E\left[A_{c} C_{\theta_{R R}} A_{c}^{T}\right] \psi\left(t_{2}\right)
$$

where $C_{\theta_{R R}}$ is the square matrix of expansion coefficients of the input's correlation function, which is given by:

$$
\theta_{R R}\left(t_{1}, t_{2}\right)=E\left[R\left(t_{1}\right) R\left(t_{2}\right)\right]=\psi\left(t_{1}\right)^{T} E\left[C_{R}\left(C_{R}\right)^{T}\right] \psi\left(t_{2}\right)=\psi\left(t_{1}\right)^{T} E\left[C_{\theta_{R R}}\right] \psi\left(t_{2}\right)=\psi\left(t_{1}\right)^{T} C_{\theta_{R R}} \psi\left(t_{2}\right)
$$

The covariance function of the system's input is defined as:

$$
\kappa_{R R}\left(t_{1}, t_{2}\right)=E\left\{\left[R\left(t_{1}\right)-m_{R}\left(t_{1}\right)\right]\left[R\left(t_{2}\right)-m_{R}\left(t_{2}\right)\right]\right\}=E\left[R\left(t_{1}\right) R\left(t_{2}\right)\right]-m_{R}\left(t_{1}\right) m_{R}\left(t_{2}\right)=\theta_{R R}\left(t_{1}, t_{2}\right)-m_{R}\left(t_{1}\right) m_{R}\left(t_{2}\right)
$$

Expanding (26) in terms of orthogonal functions gives:

$$
\kappa_{R R}\left(t_{1}, t_{2}\right)=\psi\left(t_{1}\right)^{T} C_{\kappa_{R R}} \psi\left(t_{2}\right)=\psi\left(t_{1}\right)^{T} C_{\theta_{R R}} \psi\left(t_{2}\right)-\psi\left(t_{1}\right)^{T} C_{m_{R}}\left(C_{m_{R}}\right)^{T} \psi\left(t_{2}\right)
$$

The spectral characteristics of the input signal's moments are given by:

$$
C_{\kappa_{R R}}=C_{\theta_{R R}}-C_{m_{R}}\left(C_{m_{R}}\right)^{T}
$$

Substituting (28) into (24) gives:

$$
\theta_{Y Y}\left(t_{1}, t_{2}\right)=\psi\left(t_{1}\right)^{T} E\left[A_{c} C_{\theta_{R R}} A_{c}^{T}\right] \psi\left(t_{2}\right)=\psi\left(t_{1}\right)^{T} E\left[A_{c}\left\{C_{\kappa_{R R}}+\left(C_{m_{R}}\right)\left(C_{m_{R}}\right)^{T}\right\} A_{c}^{T} \boldsymbol{\psi}\left(t_{2}\right)\right.
$$

The covariance function of the system's output is then given by:

$$
\begin{aligned}
\kappa_{Y Y}\left(t_{1}, t_{2}\right) & =\psi\left(t_{1}\right)^{T} C_{\kappa_{Y Y}} \psi\left(t_{2}\right)=\theta_{Y Y}\left(t_{1}, t_{2}\right)-m_{Y}\left(t_{1}\right) m_{Y}\left(t_{2}\right) \\
& =\psi\left(t_{1}\right)^{T} E\left[A_{c}\left\{C_{\kappa_{R R}}+\left(C_{m_{R}}\right)\left(C_{m_{R}}\right)^{T}\right\} A_{c}^{T} \boldsymbol{\psi}\left(t_{2}\right)-\psi\left(t_{1}\right)^{T} C_{m_{Y}}\left(C_{m_{Y}}\right)^{T} \psi\left(t_{2}\right)\right.
\end{aligned}
$$

or in spectral form:

$$
C_{\kappa_{Y Y}}=E\left[A_{c}\left\{C_{\kappa_{R R}}+\left(C_{m_{R}}\right)\left(C_{m_{R}}\right)^{T}\right\} A_{c}^{T}\right]-C_{m_{Y}}\left(C_{m_{Y}}\right)^{T}
$$

Eq. (31) gives the relationship between the spectral characteristics of the moments of the system's output and input.

To summarize, the system's output's mean and covariance are given by (22) and (31), respectively. If the system does not have random parameters, the closed-loop operational matrix is deterministic and Eqs. (22) and (31) reduce to:

$$
\begin{aligned}
& C_{m_{Y}}=A_{C} C_{m_{R}} \\
& C_{\kappa_{Y Y}}=A_{c}\left\{C_{\kappa_{R R}}+\left(C_{m_{R}}\right)\left(C_{m_{R}}\right)^{T}\right\} A_{c}^{T}-C_{m_{Y}}\left(C_{m_{Y}}\right)^{T}
\end{aligned}
$$


Thus,

$$
\begin{aligned}
& m_{Y}(t)=\psi(t)^{T} A_{c} C_{m_{R}} \\
& \kappa_{Y Y}\left(t_{1}, t_{2}\right)=\psi\left(t_{1}\right)^{T} A_{c}\left\{C_{\kappa_{R R}}+\left(C_{m_{R}}\right)\left(C_{m_{R}}\right)^{T}\right\} A_{c}^{T} \psi\left(t_{2}\right)-\psi\left(t_{1}\right)^{T} C_{m_{Y}}\left(C_{m_{Y}}\right)^{T} \psi\left(t_{2}\right)
\end{aligned}
$$

Random parameters result in the operational matrix $A_{c}$ in (22) and (31), and its moment can be estimated by stochastic collocation, described in the next section.

\section{Methods for quantifying uncertainties}

\subsection{Operational matrix method}

In general, a closed loop operational matrix can be given by (16) where $a_{i}$ and $b_{j}$ are independent random parameters. First, consider the decomposition

$$
a_{i}=\bar{a}_{i}+a_{i_{r}} ; \quad b_{j}=\bar{b}_{j}+b_{j_{r}}
$$

where $\bar{a}_{i}$ and $\bar{b}_{j}$ are the means of $a_{i}$ and $b_{j} ; a_{i_{r}}$ and $b_{j_{r}}$ are the random central components (i.e. zeros mean).

Using (34) and Neumann series, the operational matrix in (16) can be rewritten as [17]:

$$
\begin{aligned}
A_{c} & =\left(a_{l} D_{\alpha_{l}}+\ldots+a_{0} D_{\alpha_{0}}\right)^{-1}\left(b_{m} D_{\beta_{m}}+\cdots+b_{0} D_{\beta_{0}}\right)=\left(I+A_{L}\right)^{-1} A_{R}=\left(I+\bar{A}_{L}+A_{L_{r}}\right)^{-1} A_{R}=A_{0} \sum_{v=0}^{\infty}(-1)^{v}\left(A_{L_{r}} A_{0}\right)^{v} A_{R} \\
& =A_{0} \sum_{j=0}^{m} \sum_{v=0}^{\infty}(-1)^{v}\left(A_{L_{r}} A_{0}\right)^{v}\left(\bar{A}_{R}+A_{R_{r}}\right)=A_{0} \sum_{j=0}^{m} \sum_{v=0}^{\infty}(-1)^{v}\left(\sum_{i=1}^{l} D_{\alpha_{i}} a_{i_{e}} A_{0}\right)^{v}\left(D_{\beta_{i}} b_{j_{r}}+\bar{A}_{R}\right)
\end{aligned}
$$

with

$$
\begin{aligned}
& A_{R}=\left(b_{m} D_{\beta_{m}}+\cdots+b_{0} D_{\beta_{0}}\right) ; \quad \bar{A}_{R}=\left(\bar{b}_{m} D_{\beta_{m}}+\cdots+\bar{b}_{o} D_{\beta_{0}}\right) \\
& A_{L}=\left(a_{l} D_{\alpha_{l}}+\cdots+a_{0} D_{\alpha_{0}}\right) ; \quad \bar{A}_{L}=\left(\bar{a}_{l} D_{\alpha_{l}}+\cdots+\bar{a}_{0} D_{\alpha_{0}}-I\right) ; \quad A_{L_{r}}=\left(a_{l_{r}} D_{\alpha_{l}}+\cdots+a_{0_{r}} D_{\alpha_{0}}\right) \\
& A_{0}=\left(I+\bar{A}_{L}\right)^{-1}
\end{aligned}
$$

A truncated version of (35) is used for computing the moment of random matrices in (22) and (31), and computing the spectral characteristics of the output's mean and covariance. However, since a Neumann series is used to approximate a random matrix, this approach is inherently restricted to small uncertainties. To overcome this limitation, stochastic collocation [15] is used here and is described below.

\subsection{Stochastic collocation for operational matrices}

Stochastic collocation, briefly described below, is based on polynomial chaos and can easily estimate the means and variances of complex dynamics.

- Assume that a random operational matrix has the form:

$$
A=A(\xi)
$$

where $\xi=\left(\xi_{1}, \xi_{2}, \ldots, \xi_{n}\right)$ is a vector of independent random parameters with probability density functions (pdf) $\rho_{i}\left(\xi_{i}\right): \Gamma_{i} \rightarrow \mathbb{R}^{+}$. The joint pdf of $\boldsymbol{\xi}$ is $\boldsymbol{\rho}=\prod_{i=1}^{n} \rho_{i}$ with the support $\boldsymbol{\Gamma} \equiv \prod_{i=1}^{n} \Gamma_{i} \in \mathbb{R}^{n}$.

- For each random parameter, choose a suitable quadrature set $\left\{\xi_{i}^{(m)}, w^{(m)}\right\}_{m=1}^{q_{i}}$ according to the probability density so that one-dimensional integration can be accurately approximated by:

$$
\int_{\Gamma_{i}} A\left(\xi_{i}\right) \rho_{i}\left(\xi_{i}\right) d \xi_{i}=\sum_{i=1}^{q_{i}} A\left(\xi_{i}^{(m)}\right) w_{i}^{(m)}
$$

where $\xi_{i}^{(m)}$ is the $m$ th node and $w^{(m)}$ is the corresponding weight.

- Construct a multi-dimensional cubature set by tensorizing the one-dimensional quadrature set over all the combined multi-index $\left(j_{1}, \ldots, j_{n}\right)$. Since the manipulation of the multi-index $\left(j_{1}, \ldots, j_{n}\right)$ is cumbersome in practice, a single index is preferable for the manipulation of these equations. The multi-index is often replaced by a graded lexicographic order index $\mathbf{j}$ [15]. Since the probability density functions are the same as the weighting functions of the cubature, the moment of the random matrix is approximated by:

$$
E[A]=\int_{\Gamma} A(\xi) \boldsymbol{\rho}(\xi) d \xi=\sum_{\mathbf{j}=1}^{Q} A\left(\xi^{(\mathbf{j})}\right) \mathbf{w}^{(\mathbf{j})}=\sum_{j_{1}=1}^{q_{1}} \ldots \sum_{j_{n}=1}^{q_{n}} A\left(\xi_{1}^{\left(j_{1}\right)}, \ldots, \xi_{n}^{\left(j_{n}\right)}\right)\left(w_{1}^{\left(j_{1}\right)} \ldots w_{n}^{\left(j_{n}\right)}\right)
$$




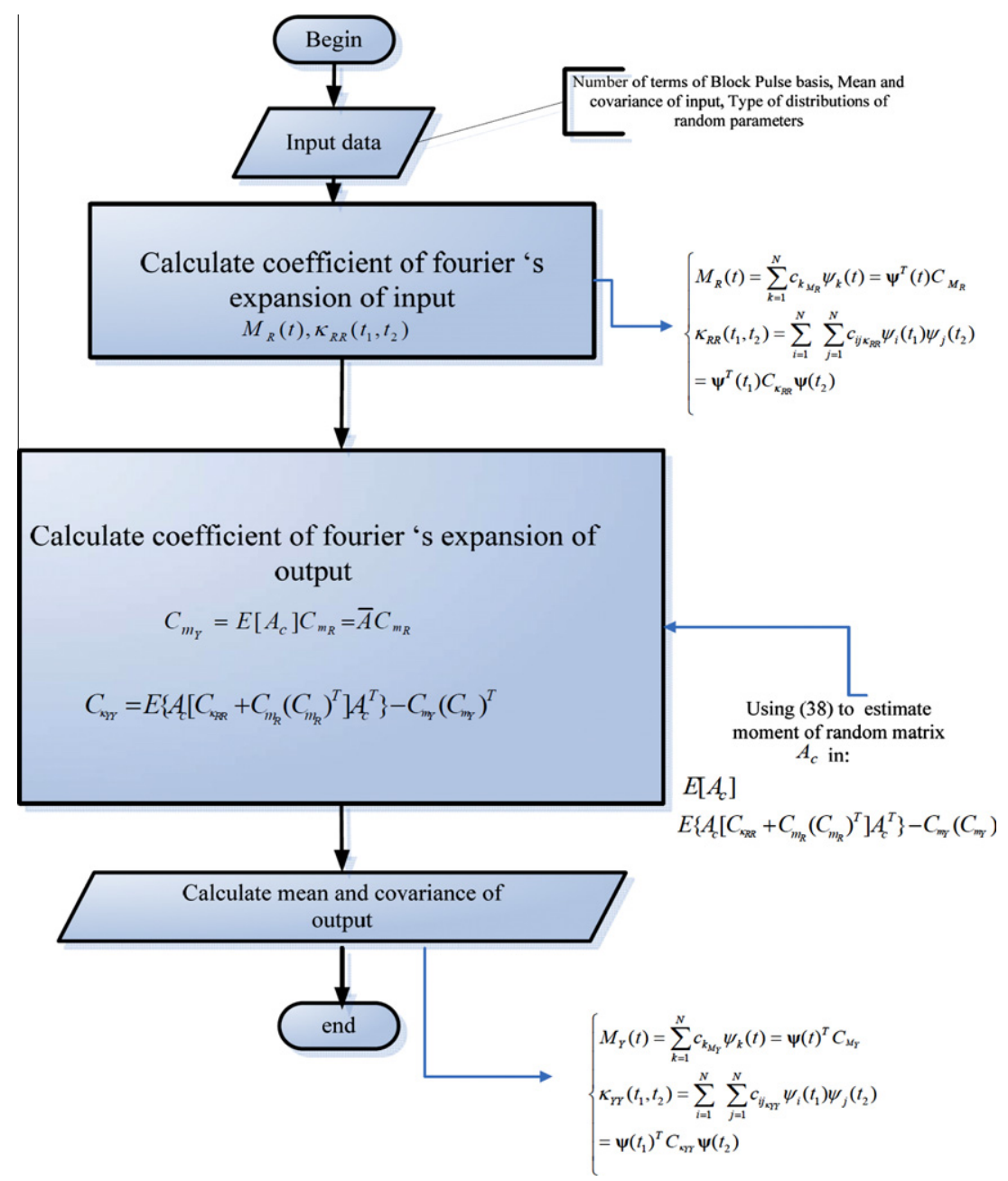

Fig. 2. Proposed numerical algorithm for calculating the mean and variance of a fractional order system output with random input and parameters.

The MATLAB suite OPQ can be used to obtain one-dimensional quadrature sets and their corresponding orthogonal polynomials (polynomial chaos) with respect to different weights [23]. The method's name is derived from the cubature nodes, which are also called collocation points. The proposed algorithm is depicted graphically in Fig. 2.

The proposed method leads to a semi-analytical relationship between the first two stochastic moments of the system's input and output and thus can significantly reduce computational times in comparison with other methods. The computational needs of other methods are discussed below.

\subsection{Karhunen-Loeve expansions}

Uncertainty quantification using either MC or gPC methods requires the finite dimensional representation of the random process. The Karhunen-Loeve (KL) method is commonly used for dimension reduction in representing random processes. Let $\mu_{X}(t)$ be the mean of random process input $X$ and $\kappa\left(t_{1}, t_{2}\right)$ be its covariance function. The KL expansion of $X$ is:

$$
X(t)=\mu_{X}(t)+\sum_{i=1}^{\infty} \sqrt{\lambda_{i}} \phi_{i}(t) \xi_{i}
$$

where $\varphi_{i}(t)$ are the eigenfunctions and $\lambda_{i}$ are the corresponding eigenvalues of the eigenvalue problem:

$$
\int_{T} \kappa\left(t_{1}, t_{2}\right) \phi_{i}\left(t_{2}\right) d t_{2}=\lambda_{i} \phi_{i}\left(t_{1}\right) \quad \text { for } t_{i} \in[-\tau, \tau]
$$

and $\left\{\xi_{i}\right\}$ are mutually uncorrelated random variables satisfying: 


$$
M\left[\xi_{i}\right]=0 ; \quad M\left[\xi_{i}, \xi_{j}\right]=\delta_{i j} ; \quad \xi_{i}=\frac{1}{\sqrt{\lambda_{i}}} \int_{T}\left(X(t)-\mu_{X}(t)\right) \varphi_{i}(t) d t, \quad \forall i
$$

For practical purposes, a finite series expansion is used, e.g.:

$$
X(t)=\mu_{X}(t)+\sum_{i=1}^{d} \sqrt{\lambda_{i}} \varphi_{i}(t) \xi_{i} d \geqslant 1
$$

What truncated order should be used in (39) depends on the decay property of the eigenvalues in the KL expansion. Fig. 3 gives decay rate of eigenvalues for a random process with exponential covariance function $\kappa_{X X}\left(t_{1}, t_{2}\right)=\exp \left(-\left|t_{1}-t_{2}\right| / a\right)$ for several correlation lengths $a$. The analytical solution of eigenvalue problem (40) is given in [15]. Fig. 3 shows that the decay rate of the eigenvalues increases with increasing correlation length. With small correlation lengths, the decay is barely visible. With a correlation length of zero, the covariance function takes the form of a Dirac delta function $\kappa\left(t_{1}, t_{2}\right)=\delta\left(t_{1}-t_{2}\right)$, and the process becomes white noise. In this case, there is no decay of the eigenvalues and hundreds of terms of the KL expansion are required for simulating white noise, leading to a system with the dimensions of random space with an order in the hundreds.

\subsection{The Monte Carlo method}

The Monte Carlo (MC) algorithm is briefly summarized as follows:

- Assume that the random process input is properly parameterized as in the previous subsection, and that the total dimension of random space is $d$, which includes random space by the finite dimensional presentation of the random process and the random parameters of the system. Generate random numbers $\xi^{(\mathbf{i})}=\left(\xi_{1}^{\left(i_{1}\right)}, \ldots, \xi_{d}^{\left(i_{d}\right)}\right)$, where $\mathbf{i}$ is the single index ordered by the graded lexicographic method, $\mathbf{i}=1, \ldots, M$ according to the given distribution.

- For each $\mathbf{i}=1, \ldots, M$, solve the governing equation (4) and obtain the solution $y\left(t, \xi^{(\mathbf{i})}\right)$.

- Estimate the required solution's statistics. For example, the solution's mean can be estimated as:

$$
\mu_{y}(t) \simeq \bar{y}(t)=\frac{1}{M} \sum_{\mathbf{i}=1}^{M} y\left(t, \xi^{(\mathbf{i})}\right)
$$

Although MC is flexible and easy to implement, its convergence is slow, i.e. $O\left(M^{-1 / 2}\right)$, where $M$ is the number of random realizations. Convergence is independent of the dimension of random space, making MC computationally tractable for systems with white noise inputs.

\subsection{The generalized polynomial chaos ( $g P C)$ method}

Stochastic collocation by gPC is briefly:

- Assume that the total dimension of random space is $d$, which includes random space by finite dimensional presentation of the random process and the random parameters of the system. Construct a suitable $d$ dimensional cubature set $\left\{\boldsymbol{\xi}^{(\mathbf{j})}, w^{(\mathbf{j})}\right\}_{\mathbf{j}=1}^{Q}$, with a suitable ordered index $\mathbf{j}$.

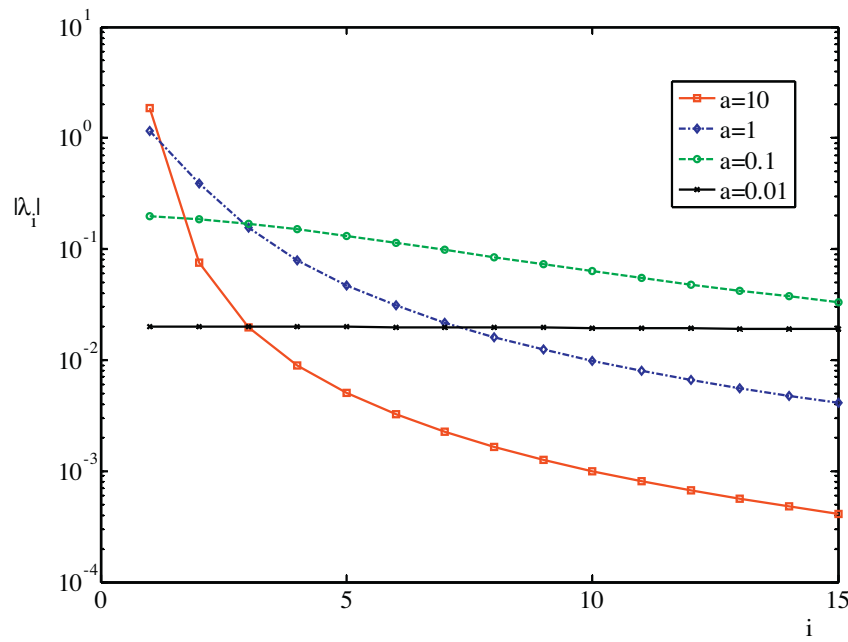

Fig. 3. The first 15 eigenvalues of the KL expansion for the exponential covariance functions with different correlation lengths $a$. 
- For each $\mathbf{j}=1, \ldots, Q$, solve (4) and obtain its solutions $y\left(t, \xi^{(\mathbf{j})}\right)$.

- The solution's mean is estimated as:

$$
\mu_{y}(t)=\sum_{\mathbf{j}=1}^{Q} y\left(t, \xi^{(\mathbf{j})}\right) \mathbf{w}^{(\mathbf{j})}=\sum_{j_{1}=1}^{q_{1}} \ldots \sum_{j_{d}=1}^{q_{d}} y\left(\xi_{1}^{\left(j_{1}\right)}, \ldots, \xi_{d}^{\left(j_{d}\right)}\right)\left(w_{1}^{\left(j_{1}\right)} \ldots w_{n}^{\left(j_{d}\right)}\right)
$$

where $q_{i}$ is the number of nodes in one dimensional quadrature.

- The variance of the solution is estimated as:

$$
D_{Y}(t) \simeq \sum_{\mathbf{j}=1}^{Q}\left(y\left(t, \xi^{(\mathbf{j})}\right)-\mu_{y}(t)\right)^{2} \mathbf{w}^{(\mathbf{j})}
$$

where $\mu_{y}(t)$ is approximated as in (44).

This algorithm shows that gPC is also almost as flexible as MC. It can solve (4) with nodes taken from the cubature and obtain statistical characteristics using the weight from the cubature set, while MC solves it using random numbers generated from the given distributions and estimates statistical characteristics as a weighted sum, with equally weights, $1 / \mathrm{M}$. gPC renders systems with low correlation length inputs as computationally intractable problems because the number of simulations is $Q=\prod_{i=1}^{d} q_{i}$ and thus rises exponentially with increasing $d$.

\section{Examples}

In this section several examples are studied to demonstrate the effectiveness of the proposed method. In Examples 1.a and 1.b, simple first order integer order systems are considered as special cases of fractional order system. Example 1.a considers a system with a first order Markov process as an input. The result by the proposed method is then compared with the results by other existing methods such as the frequency, MC and gPC methods. The advantages and disadvantages of different methods are also discussed. In Example 1.b, an integer system with ideal white noise input is examined. As discussed in Section 3, the gPC method leads to computational intractable problem for this specific input, while the proposed method still can obtain an accurate result. In the last example, a fractional order system with both parameter uncertainties and random inputs is studied to show the effectiveness of the proposed method in handling both types of uncertainties.

\subsection{Example 1: Integer order system}

\subsubsection{Example 1.a}

Consider a closed-loop system (Fig. 1) with a plant and a controller described by:

$$
G(s)=\frac{1}{s+1} ; \quad C(s)=8.16+6.31 / s
$$

The input, $R(t)$, is a Gaussian random process with mean $m_{R}(t)=0$ and covariance:

$$
\kappa_{R R}=0.25 e^{\left(-2\left|t_{1}-t_{2}\right|\right)}
$$

Note that the correlation length of this process is small because $a=0.5$.

The operational matrix for the controller is $A_{p i d}=I+6.31 A_{i}$, where $A_{i}=\left.A_{\alpha}\right|_{\alpha=1}$, and that for the plant is $A_{G}=\left(A_{i}^{-1}+I\right)^{-1}=$ $\left(A_{d}+I\right)^{-1}$. Hence, using block algebra for the operational matrices, the closed-loop operational matrix can be written as:

$$
A_{c}=\left(I+A_{G} A_{p i d}\right)^{-1} A_{G} A_{p i d}
$$

Since this system lacks random parameters, the functions describing the relation between the mean and covariance of the input and output are given by (32) and (33). Since a random input has a mean of zero, the mean output is also zero. The variance of the system's output is shown in Fig. 4; output variances estimated by MC and gPC are also shown. Since the input can be interpreted as the output of the filter $F(s)=\frac{1}{s+2}$ under the excitation of ideal white noise with a delta covariance function $\delta\left(t_{1}-t_{2}\right)$, the exact steady state variance of the output may be calculated by the frequency method as $\|F P C /(1+P C)\|_{2}^{2}$, where the \|\|$_{2}$ denotes the $\mathrm{H}_{2}$ norm of the system [24]. The simulation parameters and computational times required for each method are listed in Table 1. Fig. 4 and Table 1 show that the proposed hybrid method can give almost same accuracy for much less computational effort than the other methods. In [25], a random process input with unit correlation length was parameterized using the KL expansion with 10 terms, leading to a random space dimension of 10 . Therefore, for this system the dimension in the random space should be greater than 10 because of the decaying property of the eigenvalues described in Section 3. Non-canonical decomposition [26] can be used to reduce the dimension of the random space to 2 in this example. Hence, the random process $R(t)$ in this example is parameterized by non-canonical decomposition. Note that since the proposed hybrid method uses the algebraic relation (39) for the spectral characteristics between the input and output random moments, parameterization of the random process is not necessary. Details of the non-canonical decomposition of stationary random processes are given in Appendix A. 


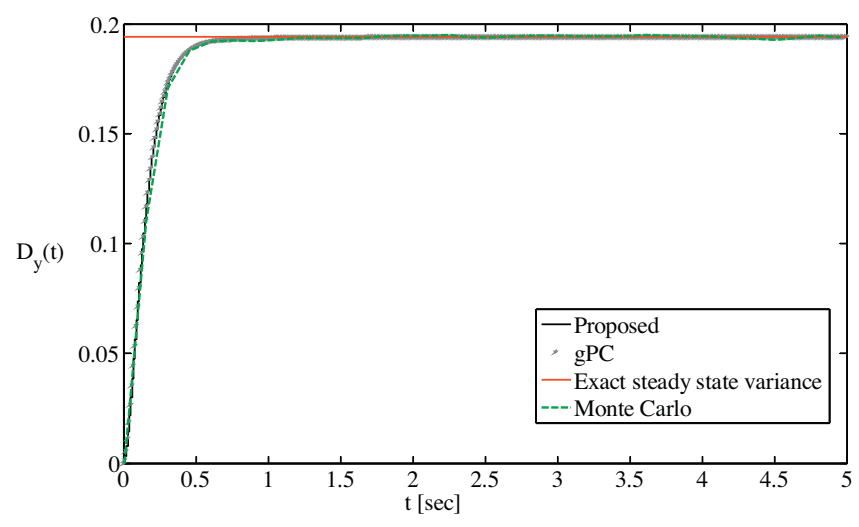

Fig. 4. Variances of the system's output in Example 1.a.

Table 1

Simulation parameters and time profiles for obtaining statistical characteristics by MC, gPC, and the proposed method in Examples 1 and 2.

\begin{tabular}{|c|c|c|c|c|c|c|}
\hline \multirow[t]{2}{*}{ Example } & \multicolumn{3}{|c|}{ Simulation parameters } & \multicolumn{3}{|c|}{ Computational time (sec.) } \\
\hline & MC & $\mathrm{gPC}$ & Proposed & MC & $\mathrm{gPC}$ & Proposed \\
\hline $1 \mathrm{a}$ & 50,000 Samples & 200 Cubature nodes & 512 Number of basis & 3147.50 & 13.47 & 0.63 \\
\hline $1 b$ & $\mathrm{~N} / \mathrm{A}$ & $\mathrm{N} / \mathrm{A}$ & 512 Number of basis & $\mathrm{N} / \mathrm{A}$ & $\mathrm{N} / \mathrm{A}$ & 0.51 \\
\hline 2 & $\mathrm{~N} / \mathrm{A}$ & 256 Cubature nodes & 512 Number of basis & $\mathrm{N} / \mathrm{A}$ & 250.01 & 4.59 \\
\hline
\end{tabular}

\subsubsection{Example 1.b}

Consider the above plant with a controller $C(s)=1 / \mathrm{s}$ where the input is ideal white noise with covariance $2 \pi \delta\left(t_{1}-t_{2}\right)=2 \pi$ $\delta(T)$ and zero mean, where $T=t_{1}-t_{2}$ and $\delta(T)$ is the Dirac delta function.

The closed loop system is $\frac{1}{s^{2}+s+1}$, and its exact variance is given as [27]:

$$
D_{Y}(t)=\frac{\pi}{2 \varsigma \omega_{n}^{3}}\left\{1-e^{-2 \varsigma \omega_{n}|t|}\left[\frac{\omega_{n}^{2}}{\omega_{d}^{2}}+\frac{\varsigma \omega_{n}}{\omega_{d}} \sin \left(2 \omega_{d} t\right)-\frac{\varsigma^{2} \omega_{n}^{2}}{\omega_{d}^{2}} \cos \left(2 \omega_{d} t\right)\right]\right\}
$$

where $\varsigma=0.5 ; \omega_{n}=1 ; \omega_{d}=\sqrt{1-\varsigma^{2}}$

The operational matrix of the closed loop system is $\left(A_{d}^{2}+A_{d}+I\right)^{-1} I$. Since the input is ideal white noise with the covariance of a singular Dirac delta function, a simple regularization method is used [28]:

$$
\delta_{\varepsilon}(\mathrm{T})= \begin{cases}k T+h & -\varepsilon \leqslant T<0 \\ -k T+h & 0<T \leqslant \varepsilon \\ 0 & |T|>\varepsilon\end{cases}
$$

Since the operational matrix uses $N$ block pulse basis functions, the integration step is $\varepsilon=\tau /(N-1)$. The approximate continuous delta function $\delta_{\varepsilon}(T)$ should satisfy:

$$
\int_{-\infty}^{\infty} \delta_{\varepsilon}(T) d T=\int_{-\varepsilon}^{\varepsilon} \delta_{\varepsilon}(T) d T=1
$$

which implies $h=\frac{N-1}{\tau} ; k=\frac{h}{\varepsilon}$. Hence, the coefficient expansion of the approximate delta covariance function becomes:

$$
C_{\kappa_{R R}}=\operatorname{diag}_{N}\left(\frac{N-1}{\tau} \mathbf{1}_{N}\right)
$$

where $\mathbf{1}_{N}$ is unit vector of size $N$ and $\operatorname{diag}(v)$ denotes the diagonal matrix where its diagonal is vector $v$.

The variance of the system predicted by the proposed method is given in Fig. 5; the exact variance is also shown. Fig. 5 and Table 1 show that the proposed method gives accurate results that closely match the analytical results without requiring great computational burden. Note that gPC leads to a computationally intractable problem, as discussed in Section 3 and it hence is not applicable to this problem. 


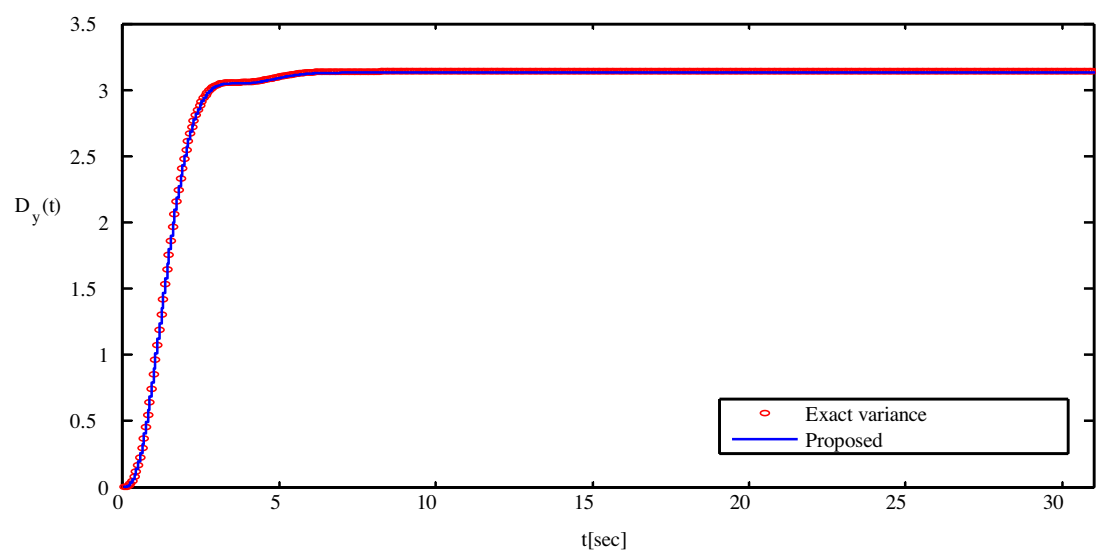

$\bar{\equiv}$

Fig. 5. Variances of the system's output in Example 1.b.

\subsection{Example 2: fractional order system}

The example above shows that both the proposed method and gPC require significantly less computation to achieve a given accuracy than traditional MC. Therefore this example only compares the proposed method and with gPC. The proposed method is used to quantify uncertainties in a closed-loop, fractional order system [29,30] with a PI $\lambda$ controller, taken from Luo et al. [31]:

$$
P(s)=\frac{k_{1}}{k_{2} S^{0.5}+1} ; C(s)=0.1817\left(1+194.4 / s^{1.216}\right)
$$

where $k_{1}$ and $k_{2}$ are uniform random variables in the interval $[0.5,1.5]$. The input $R(t)$ is a band-limited Gaussian white noise process with zero mean and a covariance function of:

$$
\kappa_{R R}=0.25 \sin c\left(\frac{t_{1}-t_{2}}{2 \pi}\right)
$$

where the sinc function is defined as:

$$
\sin c(x)=\left\{\begin{array}{l}
\sin (\pi x) /(\pi x) \text { elsewhere } \\
1 \text { for } x=0
\end{array} .\right.
$$

The operational matrix for the fractional order controller is $A_{\text {pid }}=0.1817\left(I+194.4 A_{1.216}\right)$, and for the system is $A_{G}=$ $\left(k_{2} D_{0.5}+I\right)^{-1} k_{1}$. Hence, the closed-loop operational matrix is:

$$
A_{c}=\left(I+A_{\text {pid }} A_{G}\right)^{-1} A_{\text {pid }} A_{G}
$$

Note that in this case, the closed-loop operational matrix is a random matrix due to the randomness of $k_{1}$, $k_{2}$.

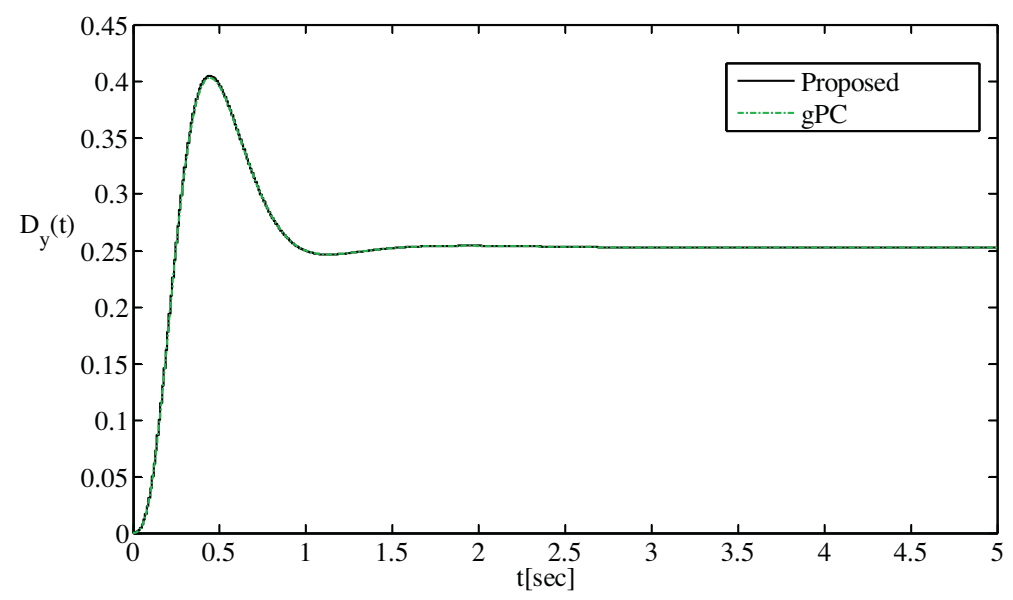

Fig. 6. Variances of the system's output in Example 2. 
The output's mean and covariance are next computed by (22), (30) and (31), in which the moment of the random matrix can be approximated by the stochastic collocation algorithm in Section 3. Again, the mean output and input are zero. The estimated variance is shown in Fig. 6, as is the variance estimated by gPC. The proposed method's advantage lies in its use of operational matrices: the output's mean and covariance are obtained directly from those of the input without any parameterization of the input. Therefore it requires a small dimension of random space, i.e. 2 (two random variables $k_{1}$ and $k_{2}$ ). In comparison gPC uses non-canonical decomposition for presenting the random input $R(t)$, which requires a higher dimension of random space, i.e. 4. Fig. 6 shows that the hybrid method can accurately predict the statistical characteristics of the system's output. For the gPC method, the MATLAB code fode_sol [21] was used to integrate the fractional order system. Simulation parameters and computational times for this example are listed in Table 1.

\section{Conclusions}

A hybrid method is proposed for the quantification of uncertainty in linear fractional order systems. By combining operational matrices and stochastic collocation, it combines each method's advantages. The use of operational matrices bypasses the computationally demanding parameterization of the random input when predicting statistical characteristics and thus reduces the dimension of the random space. The use of stochastic collocations allows handling of systems with large parameter uncertainties. Since different types of random parameters with known distributions can be associated with the method's optimal polynomial chaos and quadrature, it can easily deal with different types of random uncertainty. The use of regularization allows the proposed hybrid method to handle effectively random processes with low correlation lengths (i.e. ideal white noise). The considered examples demonstrate the proposed method's accuracy and computational efficiency for fractional order linear models over existing MC and gPC methods. However, since the operational matrix method utilizes the linear property of the system, its applicability is restricted to linear systems. Integer order systems can be treated by the proposed method simply as special cases of fractional order systems. The proposed method was examined for a PI $\lambda$ controller and a simple fractional order system but it can be easily extended for use with other, more complex, linear systems.

\section{Acknowledgement}

This work was supported by the KOSEF research grants in 2009.

\section{Appendix A. Non-canonical decomposition of stationary random processes}

Consider a stationary random process with mean $m_{X}$, covariance $\kappa_{X X}\left(t_{1}-t_{2}\right)=\kappa_{X X}(\tau)$, and variance $\sigma_{X}^{2}=\kappa_{X X}(0)$. It can be represented as [26] $Z\left(t, \xi_{1}, \xi_{2}\right)=\sigma_{X}\left(\sin \left(\xi_{2} t\right)+\xi_{1} \cos \left(\xi_{2} t\right)\right)+m_{X}$ with:

$$
E\left[\xi_{1}\right]=0 ; \quad E\left[\xi_{1}^{2}\right]=1 ; \quad f\left(\xi_{2}\right)=S_{X X}\left(\xi_{2}\right) / \sigma_{X}^{2}
$$

where $\xi_{1}$ and $\xi_{2}$ are independent, $\xi_{1}$ is Gaussian, and $\xi_{2}$ is a random variable with a probability density function (pdf) given in (57).

$Z(t)$ has a mean of:

$$
m_{Z}=E\left[Z\left(t, \xi_{1}, \xi_{2}\right)\right]=\sigma_{X} \int_{-\infty}^{\infty} \sin \left(\xi_{2} t\right) f\left(\xi_{2}\right) d \xi_{2}+m_{X}=m_{X}
$$

and a covariance function of:

$$
\begin{aligned}
E\left[\stackrel{o}{Z}\left(t_{1}\right) \stackrel{o}{Z}\left(t_{2}\right)\right] & =E\left[\sigma_{X}^{2}\left\{\sin \left(\xi_{2} t_{1}\right) \sin \left(\xi_{2} t_{2}\right)+\xi_{1}^{2} \cos \left(\xi_{2} t_{1}\right) \cos \left(\xi_{2} t_{2}\right)+\xi_{1} \cos \left(\xi_{2} t_{1}\right) \sin \left(\xi_{2} t_{2}\right)+\xi_{1} \sin \left(\xi_{2} t_{1}\right) \cos \left(\xi_{2} t_{2}\right)\right\}\right] \\
& =\sigma_{X}^{2} E\left[\sin \left(\xi_{2} t_{1}\right) \sin \left(\xi_{2} t_{2}\right)+\cos \left(\xi_{2} t_{1}\right) \cos \left(\xi_{2} t_{2}\right)\right]=\sigma_{X}^{2} \int_{-\infty}^{\infty} \cos \left(\xi_{2} \tau\right) f\left(\xi_{2}\right) d \xi_{2}
\end{aligned}
$$

where ${ }^{o} Z(t)=Z(t)-m_{Z}=Z(t)-m_{X}$ is the central component of the random process $Z(t)$. In (59), the properties of $E\left[\xi_{1}\right]=0 ; E\left[\xi_{1}^{2}\right]=1$ and the independence of $\xi_{1}, \xi_{2}$ are used to simplify the equation.

The covariance function also can be computed as the inverse Fourier transform of the power spectral density:

$$
\kappa_{Z Z}(\tau)=\kappa_{X X}(\tau)=\int_{-\infty}^{\infty} S_{X X}(\omega) e^{j \omega \tau} d \omega=\int_{-\infty}^{\infty} S_{X X}(\omega) \cos (\omega \tau) d \omega
$$

Comparing (59) and (60) gives the pdf of $\xi_{2}$ in (57). Since $\int_{-\infty}^{\infty} S_{X X}\left(\xi_{2}\right) / \sigma_{X}^{2} d \xi_{2}=1, f\left(\xi_{2}\right)$ is a proper pdf.

- A first order Markov process with mean $m_{R}$ and exponential covariance, $\kappa_{R R}(\tau)=\sigma_{X}^{2} e^{-\alpha|\tau|}$ can be parameterized as: $R=\sigma_{R}\left(\sin \left(\xi_{2} t\right)+\xi_{1} \cos \left(\xi_{2} t\right)\right)+m_{R}(t)$ where $\xi_{1}$ is Gaussian as in (57), and

$$
f\left(\xi_{2}\right)=\frac{\alpha}{\pi\left(\alpha^{2}+\xi_{2}^{2}\right)}, \xi_{2} \in(-\infty, \infty) .
$$


- Band-limited white noise with mean $m_{R}$ and covariance, $\kappa_{R R}(\tau)=2 c B_{\Omega} \sin c\left(\frac{B}{\pi} \tau\right)$ can be parameterized as: $R=\sigma_{R}(\sin$ $\left.\left(\xi_{2} t\right)+\xi_{1} \cos \left(\xi_{2} t\right)\right)+m_{R}(t)$ where $\xi_{1}$ is Gaussian as in (57), and

$$
f\left(\xi_{2}\right)=\frac{1}{2 B}, \quad \xi_{2} \in[-B, B] .
$$

\section{References}

[1] Chen W. A speculative study of 2/3-order fractional Laplacian modelling of turbulence; some though and conjectures. Chaos 2006;16:023126.

[2] Weron K, Kotulski M. On the Cole-Cole relaxation functions and related Mittag-Leffler distribution. Physica A 1996;232:180-8.

[3] Baleanu D, Guvenc ZB, Machado TJA, editors. New trend in nanotechnology and fractional calculus applications. Dordrecht: Springer; 2010.

[4] Magin RL, Akpa BS, Neuberger T, Webb AG. Fractional order analysis of Sephadex gel structure: NMR measurement reflecting anomalous diffusion. Commun Nonlinear Sci Numer Simulat, SI: Complex Syst Chaos Fraction Discont Nonlinearity 2010;16(12):4581-7.

[5] Bouafoura MK, Braiek NB. PI $\lambda \mathrm{D} \mu$ controller design for integer and fractional plants using piecewise orthogonal functions. Commun Nonlinear Sci Numer Simulat 2010;15(5):1267-78.

[6] Vinagre BM, Feliu V, Feliu JJ. Frequency domain identification of a flexible structure with piezoelectric actuator using irrational transfer functions. In: Proceedings of 37 th IEEE Conference on Decision and Control, Tampa, FL; 1998

[7] Kalges R, Radons G, Sokolov (eds) M. Anomalous transport: foundations and applications. Weinheim: Wiley VCH; 2008.

[8] Mainardi F. Fractional calculus and waves in linear viscoelasticilty. London: Imperial College Press; 2010.

[9] Oldham KB, Spanier J. Fractional calculus: theory and applications. Differentation and integration to arbitrary order. New York-London: Academic press Inc.; 1974.

[10] Miller KS, Ross B. An introduction to the fractional calculus and fractional differential equations. John Wiley and Sons; 1993.

[11] Samko SG, Kibas AA, Marichev OI. Fractional integrals and derivatives: theory and applications. Gordon and Breach; 1993.

[12] Machado JT, Kiryakova V, Mainardi F. Recent history of fractional calculus. Commun Nonlinear Sci Numer Simulat 2011;16(3):1140-53.

[13] Dunn L, Shultis JK. Exploring Monte Carlo methods. Elsevier; 2011.

[14] Pupkov KA, Egupov ND, editors. Classical and modern theory of control systems v.2. Moscow: BMSTU Press; 2003.

[15] Xiu D. Numerical method for stochastic computation: spectral approach. Princeton University Press; 2011.

[16] Xiu D, Karniadakis G. The Wiener-Askey polynomial chaos for stochastic differential equation. SIAM J Sci Comput 2003;24(2):619-44.

[17] Pupkov KA, Egupov ND, Makarenkov AM, Trofimov AI. Theory and numerical methods for studying stochastic systems. Moscow: Fizmatlits; 2003.

[18] Huang ZL, Lin XL, Lim CW, Wang Y. Statistical analysis for stochastic system including fractional derivatives. Nonlinear Dynam 2010;59:39-349.

[19] Agrawal OP. Stochastic analysis of dynamics systems contained fractional order derivative. J Sound Vib 2001;247:927-38.

[20] Agrawal OP. Analytical solution for stochastic response of fractional damped beam. J Vib Acoust 2004;126:561-6.

[21] Monje AC, Chen Y, Vingare MB, Xue D, Feliu V. Fractional-order systems and controls. London: Springer; 2010.

[22] Wang CH. On the generalization of block pulse operational matrix for fractional calculus. J Frank Inst 1983;315(2):91-102.

[23] Gaustchi W. Orthogonal polynomials: computation and approximations. Oxford University Press; 2003.

[24] Zhou K, Doyle JC. Essential of robust control. Prentice Hall; 1997.

[25] Xiu D, Tartakovsky DM. Numerical method for differential equations in random domains. SIAM J Sci Comput 2006;28(3):1167-85.

[26] Chernhecki VI. Analysis accuracy of nonlinear control systems. Masintroenhie; 1968.

[27] Sun JQ. Stochastic dynamics and control. Elsevier; 2006.

[28] Schiesser WE, Griffiths GW. A compendium of partial differential equations models: method of lines analysis with Matlab. Cambridge University Press; 2009.

[29] Gochyuk I, Hangi P. Fractional diffusion modeling of ion channel gating. Phys Rev E 2004;70:051915.

[30] Margin R. Fractional calculus in bioengineering. Begell House Publisher Inc.; 2006.

[31] Luo Y, Chen Y, Wang C, Pi G. Tuning fractional order proportional integral controllers. J Process Control 2010;20:823-83. 